\title{
Optical fluence distribution study in tissue in dark-field confocal photoacoustic microscopy using a modified Monte Carlo convolution method
}

\author{
Zhixing Xie, ${ }^{1}$ Lihong V. Wang, ${ }^{2}$ and Hao F. Zhang ${ }^{1, *}$ \\ 'Department of Electrical Engineering and Computer Science, The University of Wisconsin-Milwaukee, \\ Milwaukee, Wisconsin 53201, USA \\ ${ }^{2}$ Optical Imaging Laboratory, Department of Biomedical Engineering, Washington University in St. Louis, \\ St. Louis, Missouri 63130, USA \\ *Corresponding author: zhang25@uwm.edu
}

Received 22 August 2008; revised 7 May 2009; accepted 13 May 2009; posted 13 May 2009 (Doc. ID 100461); published 5 June 2009

\begin{abstract}
We have modified the existing convolution method of the Monte Carlo simulation for finite photon beams with both translational and rotational invariance. The modified convolution method was applied to simulate the optical fluence distribution in tissue in dark-field confocal photoacoustic microscopy. We studied the influence of the size of the dark field and the illumination incident angle on the depth position of the effective optical focus (the region with the highest fluence) and the fluence ratio (the ratio of the optical fluence at the effective optical focus inside the tissue to the optical fluence on the tissue surface along the ultrasonic axis). Within the reach of diffuse photons, the depth position of the effective optical focus increases with the size of the dark field and is much less sensitive to the incident angle. The findings show that, while the fluence at the effective optical focus decreases, the fluence ratio increases with the size of the dark field. The incident angle has a weaker influence on the fluence ratio than the size of the dark field does. An incident angle between 30 and 50 degrees gives the highest fluence at the effective optical focus. (C) 2009 Optical Society of America
\end{abstract}

OCIS codes: $170.3660,120.3890$.

\section{Introduction}

Photoacoustic (PA) imaging $[\underline{1}, 2]$ is a novel imaging modality that has been applied to in vivo imaging of tissue anatomy, tumors, burns, hemodynamic changes, and gene expression. Among all the existing PA imaging technologies, functional photoacoustic microscopy (PAM) has shown great potential in imaging subcutaneous structure and functions [ $\underline{3}-\mathbf{7}]$.

PAM employs a dark-field illumination where the weakly focused excitation laser beam has a ring-shaped cross section. This design avoids the reverberations caused by the overly strong PA signals generated from the skin surface and enables the weaker PA signals generated from deep structures

0003-6935/09/173204-08 $\$ 15.00 / 0$

(C) 2009 Optical Society of America to be detected. A high-sensitivity focused ultrasonic transducer with a large numerical aperture is employed to achieve high spatial resolution in deep tissue. In addition, the optical focus and the ultrasonic focus are designed to spatially overlap with each other in order to improve the signal-to-noise ratio (SNR) in the final image by illuminating a relatively confined region at each transducer location. At $50 \mathrm{MHz}$ ultrasonic center frequency, maximum imaging depth of more than $3 \mathrm{~mm}$ and an axial resolution of $15 \mu \mathrm{m}$ have been demonstrated. Therefore PAM is considered a high-resolution imaging modality together with confocal microscopy, two-photon microscopy, and optical coherence tomography, as they can achieve a depth-to-resolution ratio greater than 100. However, PAM offers deep penetration and optical absorption based imaging contrast that are not available from the other modalities. 
In developing and optimizing PAM, two issues must be considered carefully to achieve a good SNR and to avoid reverberation. The first issue is overlapping the focus of the ultrasonic transducer with the weakly focused laser beam. In an optically clear medium, spatial overlapping can be performed by measuring an optical absorbing object in water. The relative positions of the ultrasonic focus and geometrical optical focus are adjusted until the maximum PA signal is acquired from the object. For more detailed descriptions, please refer to [7]. However, in an optically turbid medium such as biological tissue, the highest optical fluence region may differ from the geometrical optical focus due to multiple scattering. It has been previously shown that optical focusing is very inefficient beyond one transport mean free path in a turbid medium [8]. In that case, the ultrasonic focus should ideally overlap with the region where the optical fluence is the highest (referred to as the "effective optical focus") rather than the geometrical optical focus.

The second issue is maximizing the ratio between the optical fluence at the effective optical focus inside the tissue and the optical fluence on the tissue surface along the axis of the ultrasonic detection. Here, we define such a ratio as the "fluence ratio." A high fluence ratio helps to avoid reverberation and reduce the unwanted PA signals generated from the tissue surface. As PAM employs dark-field illumination, the fluence ratio is already maximized in optically clear media because there is no optical fluence on the sample surface within the dark region directly below the ultrasonic detector [7]. However, because biological tissue has high optical scattering within the optical spectral range $(500-700 \mathrm{~nm})$ for PAM, the optical fluence on the tissue surface in the dark field cannot be ignored.

Simulation of light transport in dark-field confocal PAM is essential to understanding these two issues. The Monte Carlo (MC) method [9-12] and its extensions, such as convolution [13] and scaling [14], provide flexible tools to model light transport in turbid media in various optical imaging modalities [15-18]. The convolution method greatly reduces the computational load by decomposing a finite-size light illumination beam into pencil beams and utilizing the translational-invariance property of the impulse response (Green's function). As a result, only one actual MC simulation is necessary to acquire the overall response; however, in PAM the light incident direction rotates cylindrically with respect to the norm of the skin surface (in a spherical coordinate, a pencil beam maintains its zenith angle while varying its azimuthal angle). Although we can still decompose the finite light illumination beam into pencil beams, the impulse response for a specific pencil beam cannot be applied directly to other pencil beams in the same way as used in the existing MC convolution method. As a result, a modification is required.

Several MC studies has been reported to understand the effects of the optical scattering, optical ab- sorption, and surface roughness on the fluence distribution in a turbid medium for a solid cone focusing beam $[8,19,20]$. All the incident photon packages were tracked, and these methods can be extended to incident beams with an arbitrary shape rather than a circular shape. However, the number of photon packages required to be tracked and thus the computation load is high, especially when the size of the beam increases. Under certain regulations, a convolution based method will significantly reduce the computational load and is unlikely to be affected much by the size of the focusing beam.

In this work we modified the existing MC convolution method to be suitable for finite illumination beams with an identical incident angle and rotational incident directions. The incident angle $\varphi$ of a pencil beam was defined as the angle between the beam and the normal vector of the tissue surface (zenith angle) [Fig. 1(a)]. Besides the time invariance, the impulse response for a pencil beam was considered to be both translational and rotational invariant. The modified convolution method was then applied to simulate the light transport in dark-field confocal PAM. The influences of the illumination patterns on the above two key issues were studied.

\section{Methods}

\section{A. Impulse Responses for a Pencil Beam}

Let us assume that the Green's function of an oblique-incident pencil beam $\left(\mathrm{PB}_{i}\right)$ at the origin is

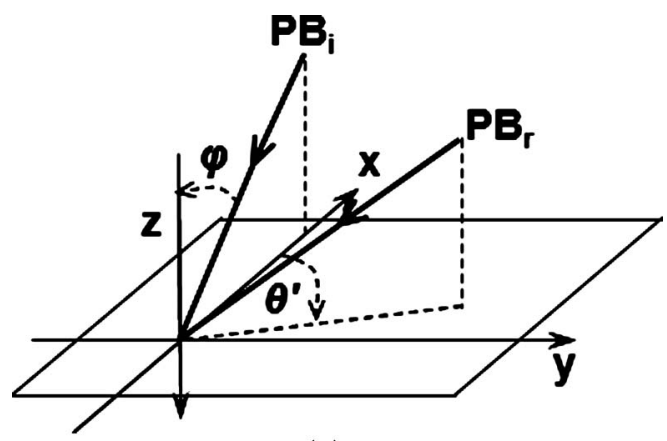

(a)

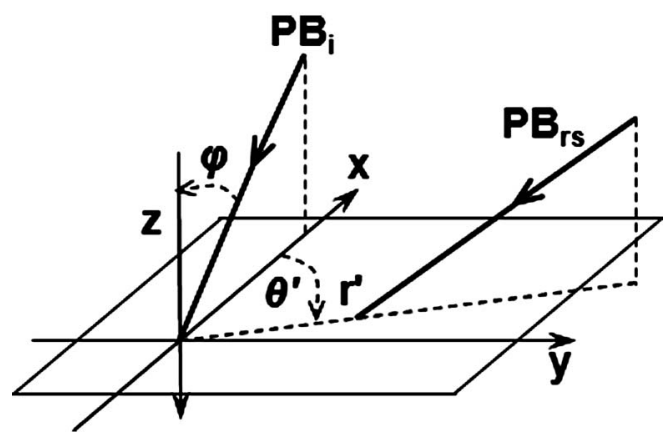

(b)

Fig. 1. Geometries of pencil beams: (a) rotation of a pencil beam; (b) rotation and translation of a pencil beam. $\mathrm{PB}_{i}$, incident pencil beam; $\mathrm{PB}_{r}$, pencil beam after rotation by $\theta^{\prime}$ from $\mathrm{PB}_{i} ; \mathrm{PB}_{r s}$, pencil beam after translation by $r^{\prime}$ from $\mathrm{PB}_{r}$. 
denoted as $G_{p}(r, \theta, z)$ in a cylindrical coordinate system and $G_{d}(x, y, z)$ in a Cartesian coordinate system. Without losing generality, the tissue surface is along the $x-y$ plane and $\mathrm{PB}_{i}$ is within the $x-z$ plane, as shown in Fig. 1(a). The relationship between the two expressions of the Green's function are

$G_{d}(x, y, z)=G_{p}\left(\sqrt{x^{2}+y^{2}}, \arctan 2(x, y), z\right)$,

$G_{p}(r, \theta, z)=G_{d}(\cos \theta, r \sin \theta, z)$,

where $r=\sqrt{x^{2}+y^{2}}, \cos \theta=x / \sqrt{x^{2}+y^{2}}, \sin \theta=y /$ $\sqrt{x^{2}+y^{2}}$, and $\arctan 2(x, y)=\arctan (y / x)+(1-$ $\operatorname{sgn}(x)) \operatorname{sgn}(y)(\pi / 2)$. Here, $\arctan 2(x, y)$ denotes the arctangent of $y / x$, taking into account which quadrant the point $(x, y)$ is in, and $\operatorname{sgn}()$ is the sign function. For the sake of conciseness, expressions in both cylindrical and Cartesian coordinates are used in the remaining derivations.

To acquire the Green's function for a new pencil beam $\left(\mathrm{PB}_{r}\right)$ that is rotated from $\mathrm{PB}_{i}$ by an angle $\theta^{\prime}$ around the $z$ axis [Fig. 1(a)], we can assume that the Green's function is rotational invariant as long as the incident angle is constant. Therefore the Green's function for $\mathrm{PB}_{r}$ can be written as

$$
\begin{aligned}
G_{p}\left(r, \theta, z: \theta^{\prime}\right) & =G_{p}\left(r, \theta-\theta^{\prime}, z\right) \\
& =G_{d}\left(r \cos \left(\theta-\theta^{\prime}\right), r \sin \left(\theta-\theta^{\prime}\right), z\right) \\
& =G_{d}\left(x^{\prime}, y^{\prime}, z\right),
\end{aligned}
$$

where $x^{\prime}=r \cos \left(\theta-\theta^{\prime}\right)$ and $y^{\prime}=r \sin \left(\theta-\theta^{\prime}\right)$.

In a more general situation, a new pencil beam $\mathrm{PB}_{r s}$ is further translated by a distance $r^{\prime}$ along the radius direction after being rotated by $\theta^{\prime}$ [Fig. 1(b)]. Under the constraint of a constant incident angle, we also can assume the translational invariance of the Green's function as described in [13]. Therefore the Green's function for $\mathrm{PB}_{r s}$ is given by

$$
\begin{aligned}
G_{d}\left(x, y, z: r^{\prime}, \theta^{\prime}\right) & =G_{d}\left(x^{\prime}, y^{\prime}, z: r^{\prime}\right)=G_{d}\left(x^{\prime}-r^{\prime}, y^{\prime}, z\right) \\
& =G_{d}\left(r \cos \left(\theta-\theta^{\prime}\right)-r^{\prime}, r \sin \left(\theta-\theta^{\prime}\right), z\right) .
\end{aligned}
$$

By combining Eq. (י) with Eq. (1), we have

$$
\begin{aligned}
G_{p}\left(r, \theta, z: r^{\prime}, \theta^{\prime}\right)= & G_{p}\left(\sqrt{r^{2}+r^{\prime 2}-2 r r^{\prime} \cos \left(\theta-\theta^{\prime}\right)},\right. \\
& \arctan 2\left(r \cos \left(\theta-\theta^{\prime}\right)-r^{\prime}\right. \\
& \left.\left.r \sin \left(\theta-\theta^{\prime}\right)\right), z\right) .
\end{aligned}
$$

Here, $G_{p}\left(r, \theta, z: r^{\prime}, \theta^{\prime}\right)$ is the Green's function for the incident pencil beam $\mathrm{PB}_{r s}$ at $\left(r^{\prime}, \theta^{\prime}\right)$.

\section{B. Finite-Size Beam Illumination}

Let us assume that a laser beam with a finite size and an intensity profile $s(x, y)$ or $s(r, \theta)$ illuminates an area $S$ on the tissue surface. The overall response
$C_{p}(r, \theta, z)$ can be obtained by integrating $G_{p}(r, \theta$, $\left.z: r^{\prime}, \theta^{\prime}\right)$ around the illumination area $S$, which is given as

$$
C_{p}(r, \theta, z)=\iint_{S} G_{p}\left(r, \theta, z: r^{\prime}, \theta^{\prime}\right) s\left(r^{\prime}, \theta^{\prime}\right) \mathrm{d} S .
$$

Figure 2(a) shows an illumination with only a translational relationship between decomposed pencil beams. Based on the shift invariance, we have

$$
G_{d}\left(x, y, z: x^{\prime}, y^{\prime}\right)=G_{d}\left(x-x^{\prime}, y-y^{\prime}, z\right)
$$

where $x^{\prime}$ and $y^{\prime}$ are arbitrary translational distances along the $x$ and $y$ axes, respectively. The combination of Eq. (ㅁ) with Eq. (1) gives

$$
\begin{gathered}
G_{p}\left(r, \theta, z: r^{\prime}, \theta^{\prime}\right)=G_{p}\left(\sqrt{r^{2}+r^{\prime 2}-2 r r^{\prime} \cos \left(\theta-\theta^{\prime}\right)},\right. \\
\left.\arctan 2\left(r \cos \theta-r^{\prime} \cos \theta^{\prime}, r \sin \theta-r^{\prime} \sin \theta^{\prime}\right), z\right) .
\end{gathered}
$$

The further combination of Eq. (ㅁ) with Eq. (ㅁ) yields

$$
\begin{aligned}
C_{p}(r, \theta, z)= & \iint_{S} G_{p}\left(\sqrt{r^{2}+r^{2}-2 r r^{\prime} \cos \left(\theta-\theta^{\prime}\right)},\right. \\
& \arctan 2\left(r \cos \theta-r^{\prime} \cos \theta^{\prime},\right. \\
& \left.\left.r \sin \theta-r^{\prime} \sin \theta^{\prime}\right), z\right) s\left(r^{\prime}, \theta^{\prime}\right) r^{\prime} \mathrm{d} \theta^{\prime} \mathrm{d} r^{\prime} .
\end{aligned}
$$

If the finite-size beam is incident normally on the $x-y$ plane and $G_{p}$ is cylindrically symmetric, Eq. ( $\left.\underline{8}\right)$ can be simplified as

$$
\begin{aligned}
C_{p}(r, \theta, z)= & \iint_{S} G_{p}\left(\sqrt{r^{2}+r^{\prime 2}-2 r r^{\prime} \cos \left(\theta-\theta^{\prime}\right)}, z\right) \\
& \times s\left(r^{\prime}, \theta^{\prime}\right) r^{\prime} \mathrm{d} \theta^{\prime} \mathrm{d} r^{\prime},
\end{aligned}
$$

which is the same as the convolution formula given in [13].

Figure 2(b) shows the optical illumination in the dark-field confocal PAM, where both the translation and rotation of a pencil beam exist. The incident direction changes within the illumination area $S$, but the incident angles for all the decomposed pencil beams are identical. By combining Eqs. (1), (ㅁ), and (ㅁ), we have 


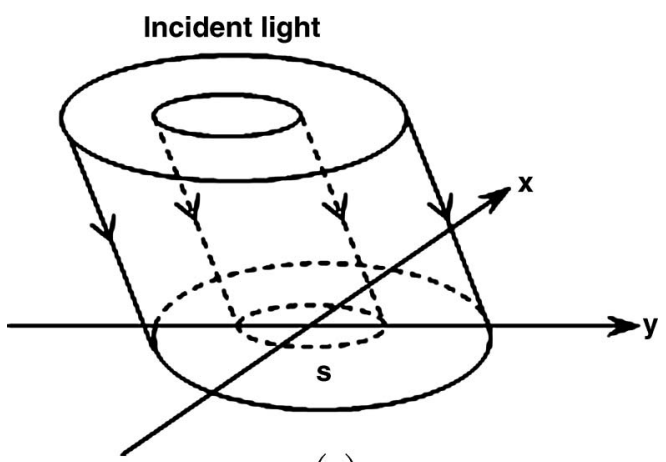

(a)

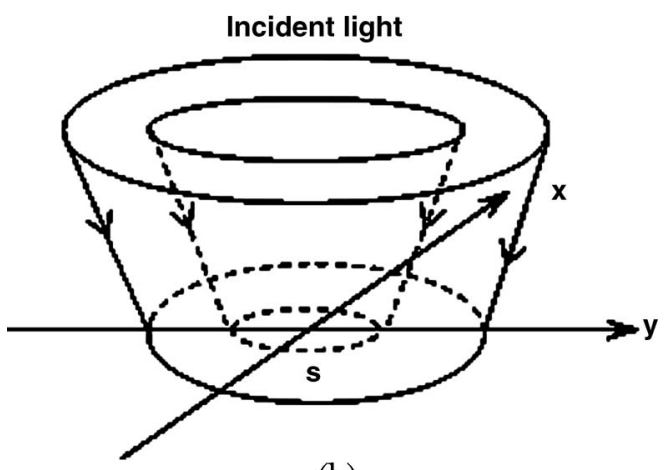

(b)

Fig. 2. Schematic of the finite-size laser beams: (a) laser beam with only parallel translation between decomposed pencil beams; (b) dark-field illumination laser beam with both rotation and translation between decomposed pencil beams.

$$
\begin{aligned}
C_{d}(x, y, z)= & \iint_{S} G_{d}\left(\frac{x x^{\prime}+y y^{\prime}-\left(x^{\prime 2}+y^{\prime 2}\right)}{\sqrt{x^{\prime 2}+y^{\prime 2}}}, \frac{y x^{\prime}-x y^{\prime}}{\sqrt{x^{\prime 2}+y^{\prime 2}}}, z\right) \\
& \times s\left(x^{\prime}, y^{\prime}\right) \mathrm{d} x^{\prime} \mathrm{d} y^{\prime}, \\
C_{p}(r, \theta, z)= & \iint_{S} G_{p}\left(\sqrt{r^{2}+r^{\prime 2}-2 r r^{\prime} \cos \left(\theta-\theta^{\prime}\right)},\right. \\
& \left.\arctan 2\left(r \cos \left(\theta-\theta^{\prime}\right)-r^{\prime}, r \sin \left(\theta-\theta^{\prime}\right)\right), z\right) \\
& \times s\left(r^{\prime}, \theta^{\prime}\right) r^{\prime} \mathrm{d} \theta^{\prime} \mathrm{d} r^{\prime} .
\end{aligned}
$$

Since the PAM illumination is cylindrically symmetrical, we have

$$
C_{p}(r, z)=C_{d}(r, 0, z),
$$

which means that only the response along the positive $x$ axis needs to be integrated. Hence, Eq. (10) is further simplified as

$$
\begin{aligned}
C_{p}(r, z)= & \iint_{S} G_{d}\left(r \times \cos \theta^{\prime}\right. \\
& \left.-r^{\prime},-r \sin \theta^{\prime}, z\right) s\left(r^{\prime}, \theta^{\prime}\right) r^{\prime} \mathrm{d} \theta^{\prime} \mathrm{d} r^{\prime} .
\end{aligned}
$$

Here, the Green's function $G_{d}(x, y, z)$ for the pencil beam at the origin is presented in the Cartesian co- ordinate system; the intensity profile $s(r, \theta)$ of the source beam and its overall response $C_{p}(r, z)$ are presented in the cylindrical coordinate system. In our simulation, the integration was carried out in cylindrical coordinates.

\section{Results and Discussions}

In this section, we present the simulation results for the two issues in dark-field confocal PAM. A twolayer skin model was used. The first layer was the epidermis, and the second layer was the dermis. The optical wavelength used in the simulation was $548 \mathrm{~nm}$, and the corresponding optical properties are given in Table 1 [9]. Because water is usually used as the acoustic coupling medium between the tissue and the ultrasonic detector in PAM experiments, the refractive indices for the top and bottom ambient media were set to 1.33 in our simulation. The thickness of the dermis for simulation purposes was set relatively larger than the anatomical values.

The Green's function for $\mathrm{PB}_{i}$ [Fig. 1(a)] was acquired from codes modified from the well known Media Center Markup Language (MCML) program, as detailed in [9]. The grid size along all axes was $0.005 \mathrm{~cm}$ in our simulation. The number of grid elements along the $x, y$, and $z$ axes were 400,200 , and 200 , respectively. Since the Green's function for $\mathrm{PB}_{i}$ is symmetric with respect to the $x-z$ plane, the 200 grid elements along the $y$ axis only covered the range from $-0.01 \mathrm{~mm}$ to $0.99 \mathrm{~cm}$. The number of photon packets traced was 30 million. In the numerical solution for Eq. (8) and (12), a 2D linear interpolation and an adaptive Simpson algorithm for quadrature with an integration tolerance of 0.0001 were used.

Before we studied the PAM, we first checked the consistency between our results and the results acquired from the published CONV (convolution) program [13] by simulating a normally incident circular top-hat beam. The top-hat beam has an energy intensity of $1 \mathrm{~mJ} / \mathrm{cm}^{2}$ at the illuminated surface and a radius of $2 \mathrm{~mm}$. The same Green's function for a pencil beam was used in both convolutions. The fluence distributions acquired from the two different programs were completely identical, and the result from our method is shown in Figs. 3(a) and 3(b).

Figures 3(a) and 3(b) show half of the fluence distribution and its contour view along a vertical $(r-z)$ cross section for the top-hat (bright-field) illumination acquired from our program. We can see that the peak fluence lies near the skin surface at a depth of $0.02 \mathrm{~cm}$ (around a transport mean free path), and it

Table 1. Optical Properties of the Two-Layer Tissue Model in the MC Simulation $^{\text {a }}$

\begin{tabular}{lccccc}
\hline Layer & $\mathrm{n}$ & $\mu_{a}\left[\mathrm{~cm}^{-1}\right]$ & $\mu_{s}\left[\mathrm{~cm}^{-1}\right]$ & $g[-]$ & Thickness $(\mathrm{cm})$ \\
\hline Epidermis & 1.34 & 0.15 & 400 & 0.85 & 0.001 \\
Dermis & 1.4 & 0.7 & 200 & 0.76 & 2 \\
\hline
\end{tabular}

${ }^{a}$ The refractive indices for the top and bottom ambient media are 1.33 . 
decays exponentially along the $z$ axis. This result indicates that if bright-field illumination is used in PAM, an overly strong PA signal generated from the skin surface will lead to a strong reverberation that overshadows PA signals generated from deeper structures.

Figures 3(c) and 3(d) show the simulated result for the fluence distribution in PAM based on the darkfield illumination [Fig. 2(b)]. For a more detailed description of the geometry of the optical illumination and ultrasonic detection, please refer to [7]. Here, the incident angle was 45 degrees, the inner radius of the illumination ring was $0.15 \mathrm{~cm}$, and the outer radius was $0.35 \mathrm{~cm}$. The energy density of the laser was $1 \mathrm{~mJ} / \mathrm{cm}^{2}$, which was homogeneously distributed within the illuminated area. Figures $3(\mathrm{c})$ and $3(\mathrm{~d})$ show that the fluence distribution follows a saddlelike profile, where the fluence at the central region is much lower than that within the illumination ring. Unlike the bright-field illumination, where the fluence peaks near the skin surface, the dark-field illumination generates a depth distribution that peaks around $0.1 \mathrm{~cm}$ below the skin surface along the $z$ axis, i.e., the effective optical focus is $0.1 \mathrm{~cm}$ below the skin surface. As a result, the signal amplitude generated from the skin surface is reduced. In comparison, the geometrical optical focus for the optical illumination was $0.15 \mathrm{~cm}$ in this situation.

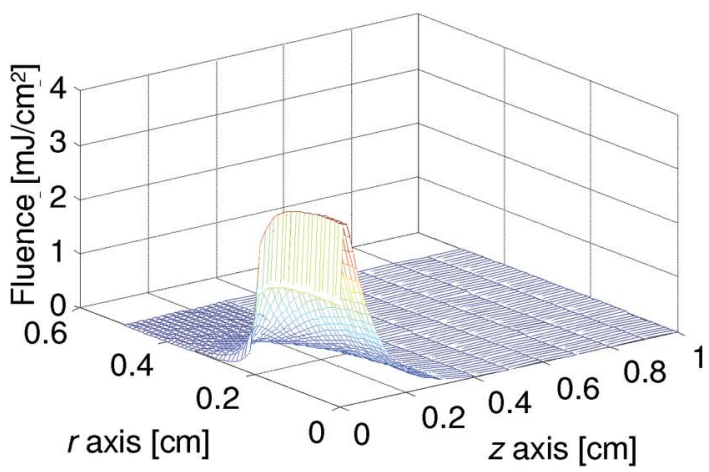

(a)

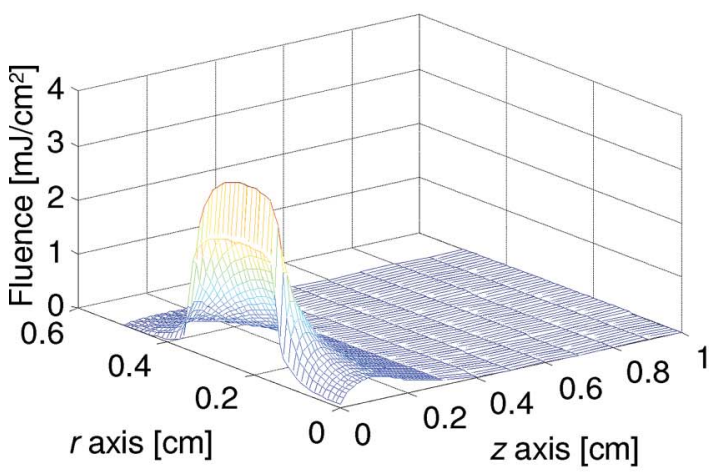

(c)
For guidance in optimizing the optical illumination in PAM, we studied the influence of (1) the incident angle and (2) the inner diameter of the illumination ring on the depth position of the effective optical focus and the fluence ratio.

First, we demonstrated here that optical scattering seriously affects the depth position of the effective optical focus in deep tissue. Figure 4(a) shows the depth-resolved dimensionless fluence distribution for an oblique-incident impulse pencil beam with a 45-degree incident angle. Such a one-dimensional fluence distribution is similar to that for a normally incident impulse pencil beam reported in a previous study [9]. Figure $4(\mathrm{~b})$ shows the contour view of the fluence distribution along the $x-y$ plane at a depth $z=0.2 \mathrm{~cm}$, which is around ten times the transport mean free path. Since the fluence distribution is symmetric with respect to the $x$ axis, we only show the positive half along the $y$ axis here. In Fig. 4(b), the location of the peak fluence shifted from the origin along the $x$ axis by a distance less than $0.04 \mathrm{~cm}$. In comparison, the geometric shift should be $0.2 \mathrm{~cm}$ at the same depth in an optically clear medium. These facts suggest that in PAM the positions of the effective optical focus and the geometrical optical focus will differ from each other conspicuously in deep tissue.

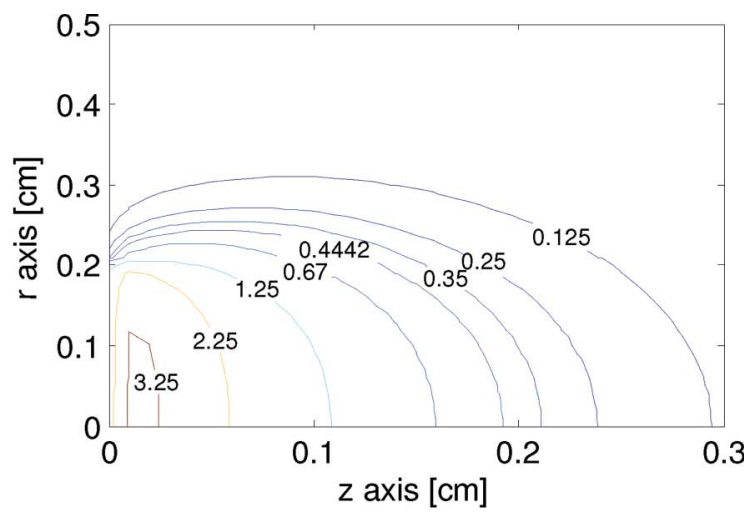

(b)

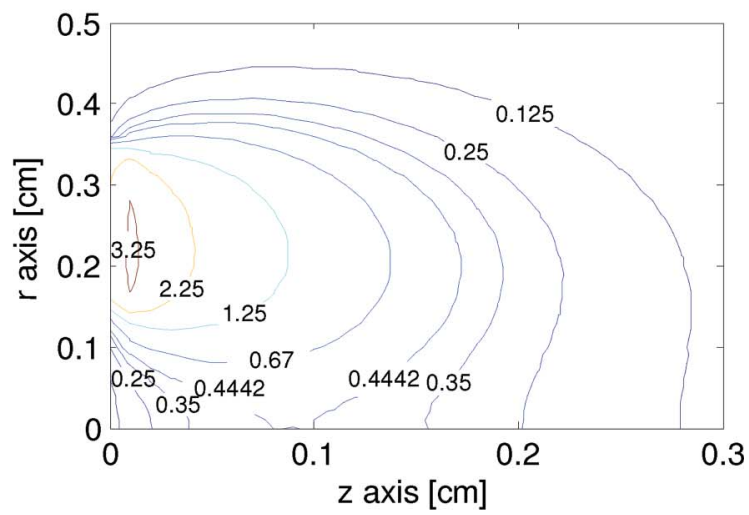

(d)

Fig. 3. (Color online) Optical fluence distributions along the $r-z$ plane for (a) bright-field and (b) dark-field optical illumination. (c), (d) Contour plots of (a) and (b), respectively. 

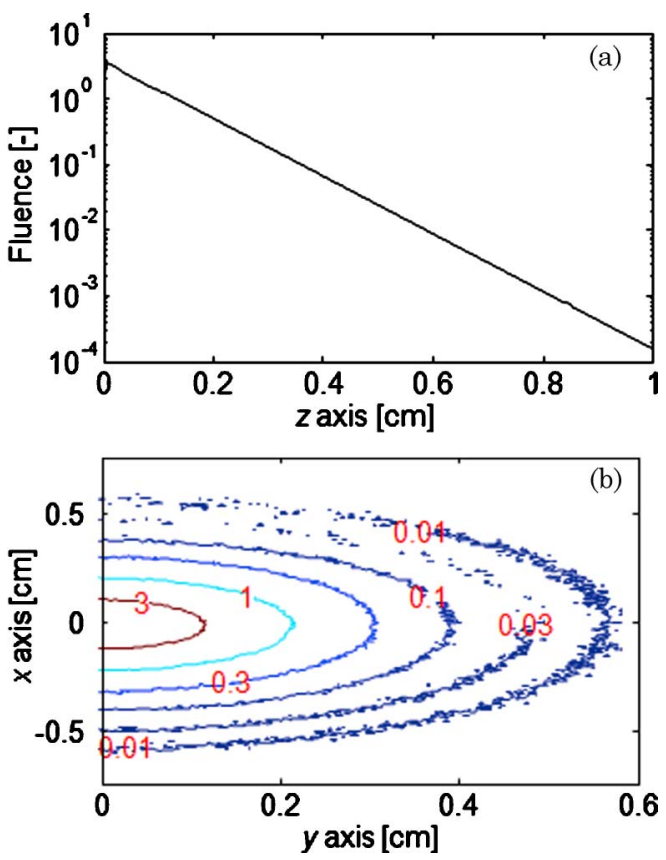

Fig. 4. (Color online) Fluence distribution for an obliquely incident pencil beam with a 45-degree incident angle: (a) depthresolved fluence distribution; fluence values on each plane that is parallel to the $x-y$ were summed; (b) fluence distribution along the $x-y$ plane at the depth $z=0.2 \mathrm{~cm}$.

In dark-field confocal PAM, the laser beam coming out of the source fiber passes through a conical lens to form a ring-shaped illumination pattern, which is then weakly focused into the tissue [7]. During data acquisition, the relative position between the conical lens and the ultrasonic detector is fixed, but the distance between ultrasonic detector and skin surface is adjustable to image the object of interest at a specific depth with the highest SNR. This situation was simulated by the obliquely and cylindrically symmetric incident finite beam with a varying inner (dark-field) radius from 0 to $0.3 \mathrm{~cm}$. The ring width was kept constant at $0.15 \mathrm{~cm}$, and the incident angle was 45 degrees. Instead of keeping a constant total energy of the finite beam, a constant energy intensity of $1 \mathrm{~mJ} / \mathrm{cm}^{2}$ at the illuminated surface was assumed. Considering that the total laser pulse energy can be changed easily by adjusting the laser system and the total pulse energy is limited only by safety regulations (maximum permissible exposure on skin surface should be less than $20 \mathrm{~mJ} / \mathrm{cm}^{2}$ in the visible spectral region) [21], the assumption was reasonable and would not affect the studies of the two issues. Moreover, both the depth position of the effective optical focus and the fluence ratio are actually independent of the incident energy.

Figure 5 shows the simulation results based on the varying inner diameter. Figure 5(a) gives the on-axis fluence at the effective optical focus and on the skin surface as a function of the inner radius. We can see here that when the dark field enlarges, the local optical fluence decreases both at the effective optical focus and on the skin surface. This result indicates that the detected PA signal amplitude decreases with increasing dark field.

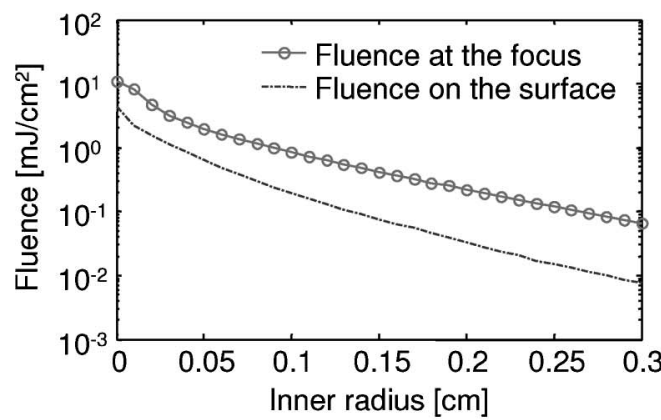

(a)

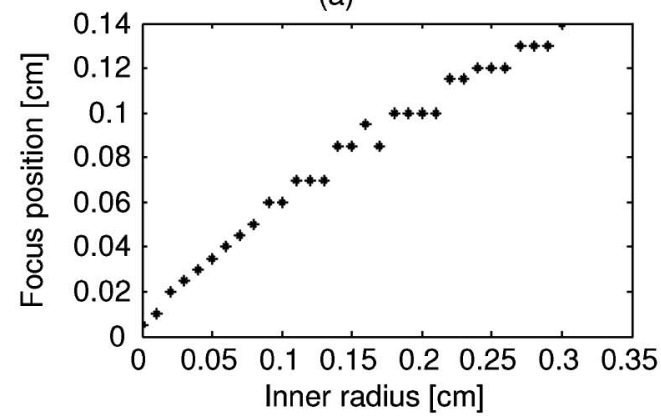

(c)

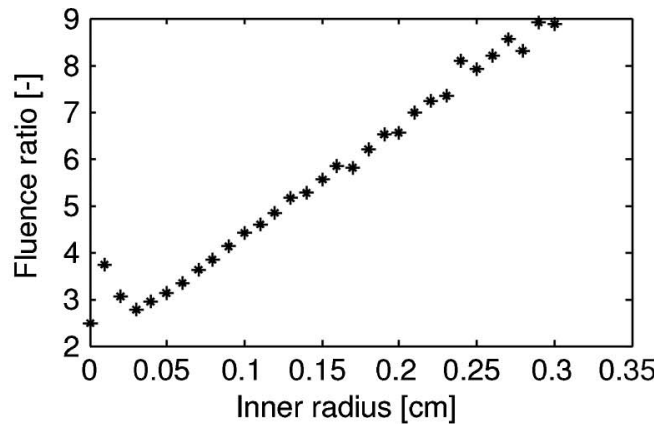

(b)

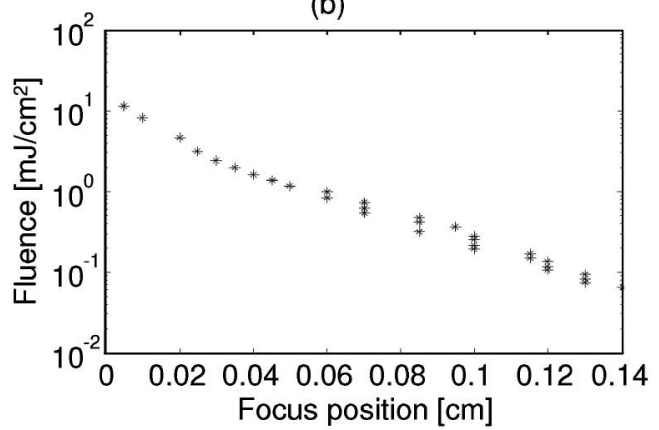

(d)

Fig. 5. Simulation results with a constant incident angle: (a) changes of optical fluence at the effective optical focus and on the skin surface as a function of the inner radius; (b) changes of the fluence ratio as a function of the inner radius; (c) changes of the depth position of the effective optical focus as a function of the inner radius; (d) changes of optical fluence at the effective optical focus when the depth position of the effective optical focus increases with the inner radius. 

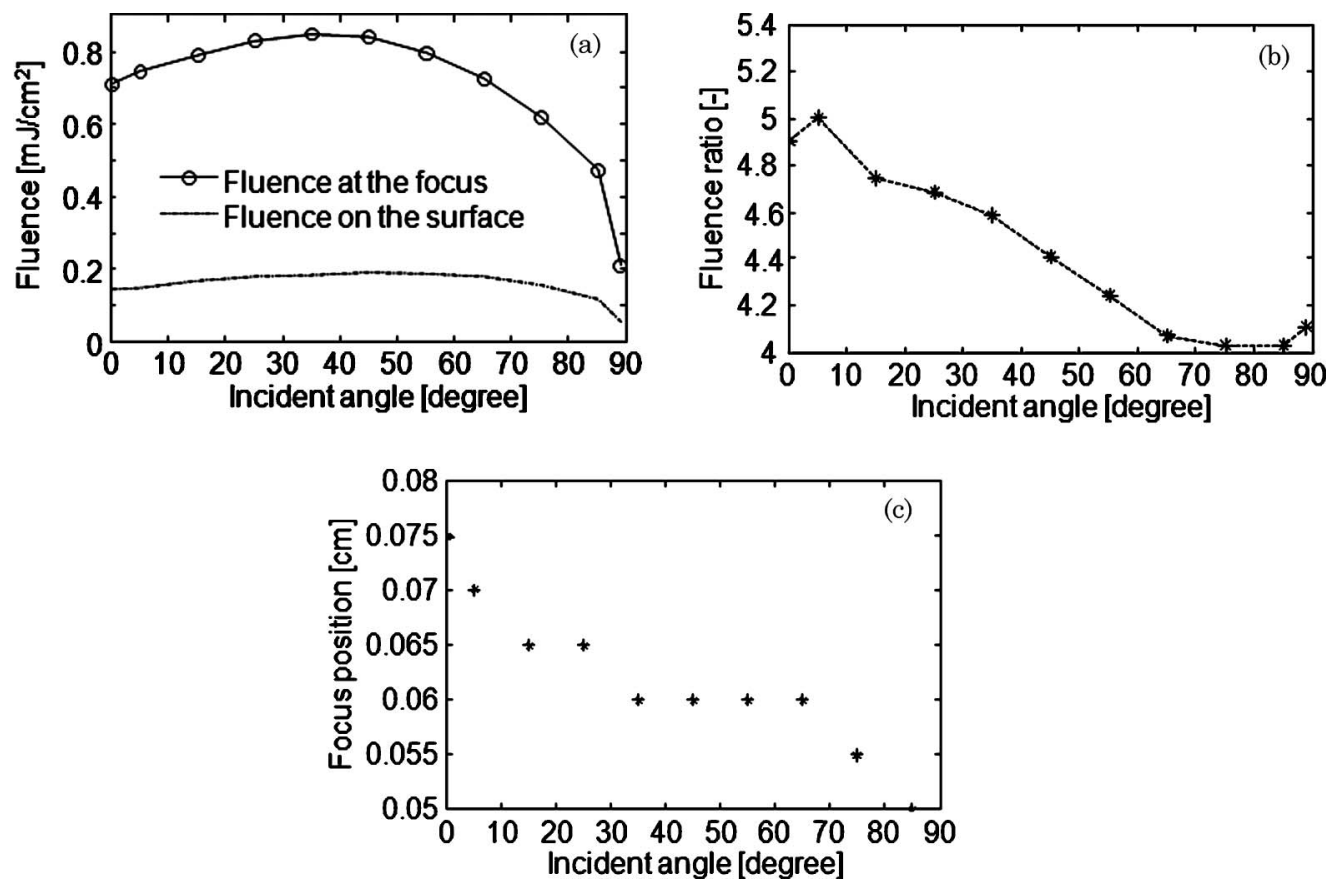

Fig. 6. Simulation results as a function of the incident angle with a fixed size of the illumination ring: (a) changes of optical fluence at the effective optical focus and on the skin surface; (b) changes of the fluence ratio; (c) changes of the depth position of the effective optical focus.

Figure $5(\mathrm{~b})$ gives the change of fluence ratio as a function of the inner radius, and Fig. 5(c) shows the depth position of the effective optical focus as a function of the inner radius. Except for a small peak around the $0.02 \mathrm{~cm}$ inner radius caused by ballistic photons within a transport mean free path, the fluence ratio increases with the inner radius. The depth position of the effective optical focus also increases with the inner radius. In Fig. 5(c), there are situations where the depth of the effective optical focus does not change with the inner radius, which is presumably caused by the lack of resolution due to the grid size.

Figure $5(\mathrm{~d})$ presents the relationship between the optical fluence at the effective optical focus and the depth position of the effective optical focus when increasing the dark field under a constant energy density on the skin surface. It shows that the local optical fluence at the effective optical focus decreases with the increase of the focus position. In fact, such a decrease in fluence value limits the maximum imaging depth of PAM, which is around 50-70 $\mathrm{mm}$ (roughly ten times the optical penetration depth) [22].

The above results suggest that increasing the dark field helps to avoid reverberation and can deliver more optical energy to deeper objects. It is important to note that in the simulation, the depth location of the geometrical optical focus ranges from 0.075 to $0.375 \mathrm{~cm}$, which is almost twice as deep as the effective optical focus herein. Thus, aligning the geometrical optical focus with the ultrasonic focus in an optically clear medium does not guarantee that the effective optical focus will accurately overlap with the acoustic focus in tissue. Our simulation may help to determine where the ultrasonic focus should be placed to maximize SNR in tissue imaging.

Next, we studied the influence of the incident angle on the fluence ratio and the depth of the effective optical focus. This was simulated with an obliquely and cylindrically symmetric incident finite beam with a fixed $0.1 \mathrm{~cm}$ inner radius and a $0.25 \mathrm{~cm}$ outer radius. The incident angle varied from 0 to 89 degrees. Again, a constant optical energy density of $1 \mathrm{~mJ} / \mathrm{cm}^{2}$ within the illuminated area was assumed.

Figure 6(a) presents the on-axis fluence values at the effective optical focus and on the skin surface as a function of the incident angle. Figures $6(\mathrm{~b})$ and 6(c) demonstrate the change of the fluence ratio and the depth position of the effective optical focus as a function of the incident angle, respectively. Although a maximum fluence is reached at the effective optical focus at an incident angle of 35 degrees, angles between 30-50 degrees show almost the same fluence level $( \pm 3 \%)$. Both the fluence ratio and the depth position of the effective focus decrease with an increasing incident angle. At an incident angle of $\sim 5$ degrees, which is nearly normally incident, the highest fluence ratio is reached. However, the fluence ratio decreases less than $20 \%$ with the incident angle increasing from 0 to 89 degrees. The depth position of the effective optical focus also has a very small change, from 0.07 to $0.05 \mathrm{~cm}$.

\section{Conclusions}

We modified the convolution method of MC simulation for finite-size photon beams with both translational and rotational invariances. The modified 
MC simulation was consistent with the CONV in simulating fluence distribution for a normally incident top-hat beam illumination. We applied the modified MC method to the dark-field confocal PAM to study the fluence distribution in tissue and to provide guidance for system optimization.

In simulating the fluence distribution, we found (1) the fluence distribution in dark-field PAM has a saddlelike shape, (2) the optical fluence is significantly reduced on the skin surface that is directly below the ultrasonic detector compared with bright-field illumination, thereby reducing the chances for reverberation, and (3) the depth position of the effective optical focus differs from that of the geometrical optical focus in deep tissue, indicating that confocal alignment only in an optically clear medium is not sufficient.

The influence of two parameters, the size of the dark field (inner radius of the illumination ring) and the illumination incident angle, on the fluence ratio and the depth position of the effective optical focus were studied. While the fluence value at the effective optical focus decreases, the fluence ratio increases with the inner radius, suggesting that a larger dark field leads to a higher imaging contrast. The depth position of the effective optical focus increases with the inner radius, suggesting that a larger dark field is required to image a deeper target. The incident angle has a relatively weaker influence on the fluence ratio. A smaller incident angle may slightly improve the imaging quality. In terms of the highest fluence at the effective optical focus, an incident angle of 30-50 degrees is optimal. Moreover, the depth position of the effective optical focus is insensitive to the incident angle.

This work was supported in part by The University of Wisconsin-Milwaukee startup fund and by a grant from The Lynde and Harry Bradley Foundation. We also thank Dr. Konstantin Maslov and Dr. Gang Yao for valuable discussions.

\section{References}

1. M. Xu and L. V. Wang, "Photoacoustic imaging in biomedicine," Rev. Sci. Instrum. 77, 041101-1-041101-22 (2006).

2. L. V. Wang, "Tutorial on photoacoustic microscopy and computed tomography," IEEE J. Sel. Top. Quantum Electron. 14, 171-179 (2008).

3. K. Maslov, G. Stoica, and L. V. Wang, "In vivo dark-field reflection-mode photoacoustic microscopy," Opt. Lett. 30, 625-627 (2005).

4. H. F. Zhang, K. Maslov, G. Stoica, and L. V. Wang, "Functional photoacoustic microscopy for high-resolution and noninvasive in vivo imaging," Nat. Biotechnol. 24, 848-851 (2006).

5. H. F. Zhang, K. Maslov, M. Li, G. Stoica, and L. V. Wang, "In vivo volumetric imaging of subcutaneous microvasculature by photoacoustic microscopy," Opt. Express 14, 9317-9323 (2006).
6. H. F. Zhang, K. Maslov, M. Sivaramakrishnan, G. Stoica, and L. V. Wang, "Imaging of hemoglobin oxygen saturation variations in single vessels in vivo using photoacoustic microscopy," Appl. Phys. Lett. 90, 053901-1-053901-3 (2007).

7. H. F. Zhang, K. Maslov, and L. V. Wang, "In vivo imaging of subcutaneous structures using functional photoacoustic microscopy," Nat. Protocols 2, 797-804 (2007).

8. L. V. Wang and G. Liang, "Absorption distribution of an optical beam focused into a turbid medium," Appl. Opt. 38, 4951-4958 (1999).

9. L.-H. Wang, S. L. Jacques, and L. Zheng, "MCML-Monte Carlo modeling of light transport in multi-layered tissues," Comput. Methods Programs Biomed. 47, 131-146 (1995).

10. S. T. Flock, M. S. Patterson, B. C. Wilson, and D. R. Wyman, "Monte-Carlo modeling of light-propagation in highly scattering tissue. 1. Model prediction and comparison with diffusion theory," IEEE Trans. Biomed. Eng. 36, 1162-1168 (1989).

11. S. T. Flock, B. C. Wilson, and M. S. Patterson, "Monte-Carlo modeling of light-propagation in highly scattering tissue. 2. Comparison with measurements in phantoms," IEEE Trans. Biomed. Eng. 36, 1169-1173 (1989).

12. S. A. Prahl, M. Keijzer, S. L. Jacques, and A. J. Welch, "A Monte Carlo model of light propagation in tissue," Proc. SPIE IS 5, 102-111 (1989).

13. L. Wang, S. L. Jacques, and L. Zheng, "CONV—convolution for responses to a finite diameter photon beam incident on multilayered tissues," Comput. Methods Programs Biomed. 54, 141-150 (1997)

14. Q. Liu and N. Ramanujam, "Scaling method for fast Monte Carlo simulation of diffuse reflectance spectra from multilayered turbid media," J. Opt. Soc. Am. A 24, 1011-1025 (2007).

15. M. C. Skala, G. M. Palmer, K. M. Vrotsos, A. GendronFitzpatrick, and N. Ramanujam, "Comparison of a physical model and principal component analysis for the diagnosis of epithelial neoplasias in vivo using diffuse reflectance spectroscopy," Opt. Express 15, 7863-7875 (2007).

16. A. Wang, V. Nammalvar, and R. Drezek, "Targeting spectral signatures of progressively dysplastic stratified epithelia using angularly variable fiber geometry in reflectance Monte Carlo simulations," J. Biomed. Opt. 12, 044012-1-04401214 (2007).

17. D. Arifler, C. MacAulay, M. Follen, and R. Richards-Kortum, "Spatially resolved reflectance spectroscopy for diagnosis of cervical precancer: Monte Carlo modeling and comparison to clinical measurements," J. Biomed. Opt. 11, 064027-1064027-16 (2006).

18. R. K. Wang, "Signal degradation by multiple scattering in optical coherence tomography of dense tissue: a Monte Carlo study," Phys. Med. Biol. 47, 2281-2299 (2002).

19. Z. Song, K. Dong, X. H. Hu, and J. Q. Lu, "Monte Carlo simulation of converging laser beams propagating in biological tissue," Appl. Opt. 38, 2944-2949 (1999).

20. J. Q. Lu, X. H. Hu, and K. Dong, "Modeling of the rough-interface effect on a converging light beam," Appl. Opt. 39, 5890-5897 (2000).

21. "American national standard for safe use of lasers," ANSI Standard Z136.1-2007 (Laser Institute of America, 2007).

22. L. V. Wang, "Prospects of photoacoustic tomography," Med. Phys. 35, 5758-5767 (2008). 